\title{
Dyspnea Associated with Silicone Breast Implant Rupture
}

\author{
Katherine Rider, Hatice Kaya, Guillermo Gutierrez \\ Department of Pulmonary, Critical Care and Sleep Medicine, The George Washington University MFA, Washington DC
}

\begin{abstract}
We present a case of a 48-year-old woman with a significant history of atopy. She presented with a 1-month history of dyspnea on exertion. Pulmonary function testing was normal, with no obstruction or reversibility post-bronchodilation. Bilateral breast implant rupture was detected on further investigation for a left upper lobe nodule, and the patient underwent bilateral implant removal. There was an improvement in her respiratory symptoms. Systemic symptoms, labeled as "Autoimmune/Inflammatory Syndrome Induced by Adjuvants," are known to be associated with breast implants, with 14\% of women in a cohort developing autoimmune disease secondary to their implants. An improvement in symptoms has been demonstrated following breast implant removal.
\end{abstract}

Keywords: Breast implants, dyspnea, hypersensitivity, rupture

Received Date: 09.01.2015 Accepted Date: 28.02.2015 Available Online Date: 28.04.2015

Address for correspondence Katherine Rider

E-mail: katherine.flower@hotmail.com

cC) (1) This work is licensed under a Creative 4.0 International License.

DOI: $10.5152 /$ ejp.2015.83803

-Available online at www.eurasianjpulmonol.com

\section{INTRODUCTION}

Silicone breast implants are known to be associated with the presence of non-specific systemic symptoms such as myalgia, fatigue, and dyspnea and can induce a syndrome which has been coined the "Autoimmune/Inflammatory Syndrome Induced by Adjuvants." Removal of silicone breast implants can result in the improvement in symptoms in a majority of women.

\section{CASE PRESENTATION}

A 48-year-old woman, with a significant history of allergy to multiple agents such as mould, fish, and alcohol, presented to the Pulmonary Clinic with a 1-month history of chest tightness associated with dyspnea on exertion. She had no significant family history of atopy and was denied occupational or environmental exposures. Her previous medical history consisted of chronic sinusitis, retinal detachment, bilateral breast enlargement, and nasal polyps with polypectomy. She had a 25 pack-year smoking history.

On examination, she was normotensive and not tachypneic. Her oxygen saturations were $96 \%$ on room air. Lung examination was normal. She was commenced on an albuterol metered-dose inhaler (MDI) as required, and pulmonary function testing (PFTs) and a chest X-ray were performed. PFTs were normal, with no obstruction and no response to bronchodilation, with normal lung volumes and diffusing capacity of the lungs for carbon monoxide (DLCO). The patient had a positive methacholine challenge test at $4 \mathrm{mg} / \mathrm{mL}$ metacholine with a $22 \%$ decrease in $\mathrm{FEV}_{1}$. There was a left upper lobe nodule on chest X-ray; therefore, a chest computed tomography (CT) scan was performed, which showed a calcified granuloma in the left upper lobe, enlarged bilateral axillary lymphadenopathy, and an enlarged left internal mammary lymph node of uncertain significance. A positron emission tomography (PET) scan showed no uptake in the granuloma but showed mild hypermetabolic circumferential breast parenchyma around the breast implants bilaterally (Figure 1). These findings were suggestive of mild intracapsular rupture with reactive adenopathy. A breast magnetic resonance imaging (MRI) scan confirmed these findings, with an evidence of bilateral intracapsular rupture with silicone migration to a left intramammary lymph node and bilateral axillary lymph nodes. There was no evidence of breast cancer. The patient underwent bilateral removal and replacement of her breast implants, with significant improvement in her respiratory symptoms. 


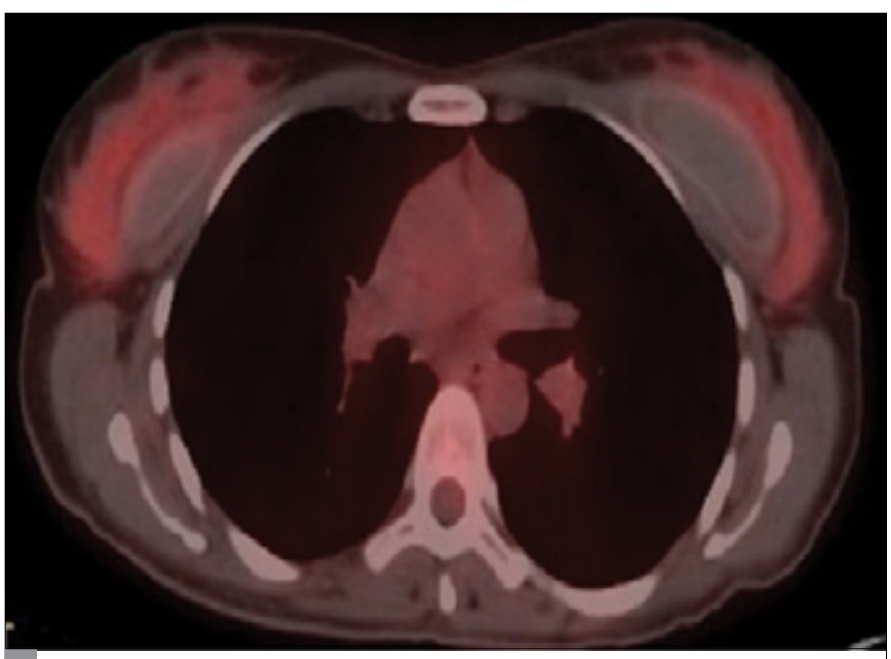

Figure 1. PET scan showing mild hypermetabolic circumferential breast parenchyma around the breast implants bilaterally PET: positron emission tomography

\section{DISCUSSION}

This case highlights the importance for physicians to consider silicone breast implant rupture in women presenting with symptoms of dyspnea. In a small case series, eight breast implant patients had findings consistent with asthma, with normal PFTs and a positive metacholine challenge test, similar to those in our patient. It was hypothesized that hexachloroplatinate, a potent sensitizer and component of breast implants, could be the likely primary etiologic agent based on findings that were consistent with platinosis in these patients (1).

Our case is also similar to women in a Dutch cohort, in which $45 \%$ of the women with breast implant rupture presented with symptoms of dyspnea. Of the women who underwent implant removal, 69\% reported improvement in their symptoms (2). They also noted that most of these women had pre-existent allergies, similar to the patient in this case.

Silicone breast implants have been used for cosmetic and reconstructive purposes since their introduction by Dow Corning in 1962 (3). Current devices are widely considered to be safe, with the overall implant rupture rate $3.5 \%$ at 6 years for Inamed implants and $0.9 \%$ at 3 years for Mentor implants $(4,5)$. However, breast implants may induce a persistent foreign body reaction that has been suggested to result in autoimmune and connective tissue disease, particularly rheumatoid arthritis and systemic sclerosis $(6,7)$.

Vasey et al. (8) found a significant increase in body aches, joint pain, myalgia, and decreased cognition in patients exposed to silicone; this finding was substantiated by Hajdu et al. (9).

The term "ASIA: Autoimmune/Inflammatory Syndrome Induced by Adjuvants" was coined in 2011 to describe the spectrum of immune-mediated diseases as the result of adjuvants such as chronic silicone exposure (10).

There have also been case reports of patients presenting with symptoms of type IV hypersensitivity to breast implants (11). In this delayed reaction, symptoms typically develop from 48 hours to several weeks after antigen exposure. Type IV hypersensitivity reactions may also develop into granuloma formation, which can then be demonstrated on lymph node biopsy.
In a small study of patients receiving silicone implants, patients demonstrated strong capsular binding of $\lg G$ and weak capsular binding of IgM. Serum lgE levels were also noted to be higher in patient sera than control sera (12). The authors concluded that silicone materials do lead to an immune response consisting of anti-silicone antibodies, which is most evident immediately adjacent to the implant itself.

\section{CONCLUSION}

Although the association between ruptured breast implants and systemic symptoms is well reported, to the authors' knowledge, there are no cases of dyspnea on exertion and wheeze because of breast implant rupture that improves on implant removal. Physicians should consider the possibility that silicone breast implants may be the cause of dyspnea.

Informed Consent: Written informed consent was obtained from the patient who participated in this case.

Peer-review: Externally peer-reviewed.

Author contributions: Concept - K.R., H.K., G.G.; Design - K.R., H.K., G.G.; Supervision - K.R.; Materials - G.G.; Data Data Collection and/or Processing - K.R., H.K., G.G.; Analysis and/or Interpretation - K.R., H.K., G.G.; Literature Search K.R.; Writing - K.R., H.K., G.G.; Critical Reviews - K.R., H.K., G.G.

Conflict of Interest: No conflict of interest was declared by the authors.

Financial Disclosure: The authors declared that this study has received no financial support.

\section{REFERENCES}

1. Harbut MR, Churchill BC. Asthma in patients with silicone breast implants: Report of a case series and identification of hexachloroplatinate contaminant as a possible etiologic agent. Isreal J Occup Health 1999; 3: 73-82.

2. Maijers $M$, de Blok $C$, Niessen $F$, van der Veldt $A$, Ritt $M$, Winters $H$, et al. Women with silicone breast implants and unexplained systemic symptoms: a descriptive cohort study. Neth J Med 2013; 71: 534-40.

3. Glicenstein J. History of augmentation mammaplasty. Ann Chir Plast Esthet 2005; 50: 337-49. [CrossRef]

4. Spear SL, Murphy DK, Slicton A, Walker PS; Inamed Silicone Breast Implant U.S. Study Group. Inamed silicone breast implant core study results at 6 years. Plast Reconstr Surg 2007; 120: 8S-16S. [CrossRef]

5. Cunningham $B$. The mentor core study on silicone memory gel breast implants. Plast Reconstr Surg 2007; 120: 19S-29S. [CrossRef]

6. Levy $Y$, Rotman-Pikielny $P$, Ehrenfeld $M$, Shoenfeld Y. Silicone breast implantation-induced scleroderma: description of four patients and a critical review of the literature. Lupus 2009; 18: 1226-32. [CrossRef]

7. Costenbader KH, Gay S, Riquelme ME, laccarino L, Doria A. Genes, epigenetic regulation and environmental factors: which is the most relevant in developing autoimmune diseases? Autoimmun Rev 2012; 11:604-9. [CrossRef]

8. Vasey FB, Zarabadi SA, Seleznick M, Ricca L. Where there's smoke there's fire: the silicone breast implant controversy continues to flicker: a new disease that needs to be defined. J Rheumatol 2003; 30: 2092-4.

9. Hajdu SD, Agmon-Levin N, Shoenfeld Y. Silicone and autoimmunity. Eur J Clin Invest 2011; 41: 203-11. [CrossRef]

10. Perricone C, Colafrancesco S, Mazor R, Soriano A, Agmon-Levin N, Shoenfeld $Y$. Autoimmune/inflammatory syndrome induced by adjuvants (ASIA) 2013: Unveiling the pathogenic, clinical and diagnostic aspects. J Autoimmun 2013; 47: 1-16. [CrossRef]

11. Dargan D, McGoldrick C, Khan K. Type IV hypersensitivity to a textured silicone breast implant. J Plast Reconstr Aesthet Surg 2012; 65: 969-72. [CrossRef]

12. Bekerecioglu M, Onat AM, Tercan M, Buyukhatipoglu H, Karakok M, Isik D, et al. The association between silicone implants and both antibodies and autoimmune diseases. Clin Rheumatol 2008; 27: 147-50. [CrossRef] 